\title{
L- and S-band Polarimetric Synthetic Aperture Radar on Chandrayaan-2 mission
}

\author{
Deepak Putrevu*, Sanjay Trivedi, Anup Das, Dharmendra Pandey, \\ Priyanka Mehrotra, S. K. Garg, Venkata Reddy, Shalini Gangele, \\ Himanshu Patel, Devendra Sharma, R. Sijwali, Nikhil Pandya, Amit Shukla, \\ Gaurav Seth, V. M. Ramanujam and Raj Kumar \\ Space Applications Centre, Ahmedabad 380 015, India
}

Dual-frequency Synthetic Aperture Radar (SAR) operating in $L$ - and $S$-band frequencies is one of the primary payloads of the Chandrayaan-2 orbiter. This payload with the capability of imaging in dual frequency (L-band: $24 \mathrm{~cm}$ wavelength and S-band: $12 \mathrm{~cm}$ wavelength) with full polarimetric mode aims for unambiguous detection, characterization and quantitative estimation of water-ice in permanently shadowed regions over the lunar poles. The payload will address the ambiguities in interpreting high values of circular polarization ratio associated with water-ice observed during previous missions to the Moon through imaging in dual-frequency fully polarimetric SAR mode. Various improved system features such as wide range of resolutions and incidence angles, synchronized $L$ and S-band operations, radiometer mode, are built into the instrument to meet the required science objectives, adhering to stringent mission requirements of low mass, power and data rates. Major scientific objectives of dual-frequency polarimetric SAR payload are: unambiguous detection and quantitative estimation of lunar polar water-ice; estimation of lunar regolith dielectric constant and surface roughness; mapping of lunar geological/morphological features and polar crater floors at high-resolution, and regional-scale mapping of regolith thickness and distribution.

Keywords: Circular polarization ratio, dual frequency, lunar polar water-ice, synthetic aperture radar.

THE possible existence of water-ice in the polar cold traps of the Moon has been strongly supported by recent studies using Mini-SAR ${ }^{1-4}$ and Moon Mineralogical Mapper (M3) $)^{5}$ of Chandrayaan-1 - the first Indian lunar mission (2008) and Mini-RF ${ }^{6}$ of Lunar Reconnaissance Orbiter (2009). The Mini-SAR and Mini-RF results are based on circular polarization ratio (CPR); a high CPR $(>1)$ is taken as an indicator of the presence of polar water-ice in permanently shadowed regions (PSRs) of the

*For correspondence. (e-mail: dputrevu@sac.isro.gov.in)
Moon. However, CPR could also be elevated due to wavelength-scale surface roughness and can lead to ambiguities in inferring the presence of polar water-ice, which could be overcome partly by the combined use of CPR and polarimetric parameters ${ }^{4}$, and modelling of the return signal. In order to unambiguously detect the presence of water-ice and quantify the same, dual-frequency (L- and S-band) fully polarimetric Synthetic Aperture Radar (SAR) was conceptualized and developed for India's second lunar mission, viz. Chandrayaan-2, launched on 22 July 2019. In addition, the polarimetric SAR will enable a host of other applications like understanding the scattering characteristics of the lunar surface and subsurface features; mapping the lunar craters and other geological/geomorphological features, especially in the polar regions with finer details, and retrieving lunar surface parameters like dielectric constant and regolith surface roughness.

The S-band imaging system of dual-frequency SAR will be useful for continuity of data from earlier MiniSAR, Mini-RF and Earth-based observations in the same band. In addition, S-band full polarimetric and highresolution data would aid in the characterization of lunar regolith morphological features and unambiguous detection of polar water-ice. The orbital L-band polarimetric SAR, being utilized for the first time for lunar studies, will enable deeper penetration into the lunar regolith and extract information from subsurface features. Simultaneous imaging in the L- and S-bands will aid quantitative estimation of water-ice.

\section{Science requirements}

\section{Unambiguous detection and quantitative estimation of water-ice}

Previous observations from Earth-based radio-telescopes and spaceborne radar instruments suggest permanently shadowed polar regions as potential zones for the presence of water-ice on the Moon. CPR, a ratio between same sense circular (SC) polarization and opposite sense 

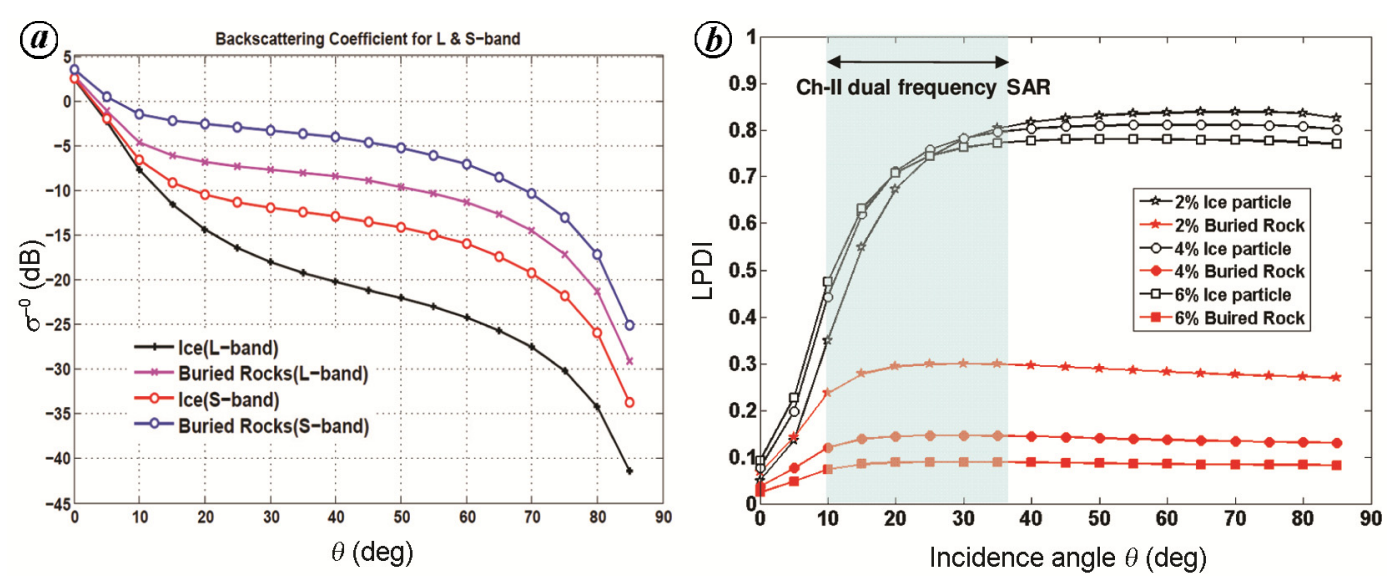

Figure 1. $\boldsymbol{a}$, Simulation results integral equation methods (IEM) showing synthetic aperture radar (SAR) backscatter in $\mathrm{HH}$-polarization in L- and S-bands over ice in regolith and buried rocks as a function of incident angle in the lunar surface conditions. $\boldsymbol{b}$, Simulated curve showing variation of lunar polar ice detection index for bedrock + regolith mixed with rock particles of different fraction and bedrock + regolith mixed with ice particles of different fraction with respect to the incident angle.

circular (OC) polarization, an important parameter derived from the radar measurements, has been traditionally used to detect the presence of water-ice in the lunar polar regions ${ }^{3,4,7}$. However, it is also realized that CPR alone cannot confirm the presence of water-ice. The complete scattering mechanism of the targets showing high CPR has to be studied, as surface roughness effect also enhances the CPR value. In this regard, polarimetric SAR data are likely to help in ascertaining precise locations of water-ice deposits in the polar regions. Integral equation methods (IEM)-based vector radiative transfer models ${ }^{8}$ have been parameterized to simulate polarimetric scattering from lunar regolith, which provides rather unambiguous information on the location of water-ice deposits through suitable inversion methods.

In-house modelling and simulation studies have revealed the impact of differences in dielectric constant $(\varepsilon)$ values of ice, lunar regolith and lunar rocks on the backscattering coefficients $\left(\sigma^{0}\right)$ at L- and S-band frequencies. Multi-layer scattering model (using IEM) has been developed to estimate the backscattering coefficients corresponding to various compositions of lunar materials in the L- and S-bands at different incident angles ${ }^{9-11}$. The model shows distinct characteristics for simulated $\mathrm{HH}-$ polarization sigma naught $(\mathrm{dB})$ corresponding to ice and buried rocks at different incident angles in the L- and Sbands (Figure 1a). The curves show buried ice and buried rocks are separable in terms of their radar crosssection (RCS) at incident angles greater than $10^{\circ}$. This characteristic has been used to develop the lunar polar-ice detection index (LPDI) corresponding to $\mathrm{HH}$ polarization as

$$
\mathrm{LPDI}=\frac{\sigma_{\mathrm{S}-\mathrm{HH}}^{0}-\sigma_{\mathrm{L}-\mathrm{HH}}^{0}}{\sigma_{\mathrm{S}-\mathrm{HH}}^{0}+\sigma_{\mathrm{L}-\mathrm{HH}}^{0}} .
$$

Simulated results show that the index can be used to separate a uniform mixture of rock + regolith and ice + regolith at different fractions over the bedrock of higher $\varepsilon$ values (Figure $1 b$ ).

SAR imaging with simultaneous L- and S-bands in single or dual polarization modes will be required for LPDI computation. This index, along with prior knowledge of dielectric constant and surface roughness estimates over the regions of interest, will be useful for volumetric estimation of polar water-ice.

\section{Dielectric constant and surface roughness estimation}

Dielectric constant and surface roughness representing concentration of radar wavelength-scale rock fragments in the lunar regolith are two important parameters that determine radar backscattering from different targets and material present on the lunar surface, and provide valuable information to feed to the volumetric estimation of water-ice, as described earlier. This information is also extremely valuable for studies about the origin and evolution of lunar features. Various empirical/semi-empirical models have been used for dielectric constant estimation of planetary bodies. Campbell et al ${ }^{12}$ suggested a dielectric inversion model for rock-poor mantling dust based on the normalized ratios between the horizontal and vertical backscattering coefficients

$$
\varepsilon_{\min }=\left(\sin \phi / \sin \left[\cos ^{-1}\left(\frac{\sigma_{\mathrm{HH}}^{0}}{\sigma_{\mathrm{VV}}^{0}}\right)^{0.25}-\phi\right]\right)^{2},
$$

where $\phi$ is the radar incident angle. The inversion model was applied on Chandrayaan-1 Mini-SAR S-band data 
over regions of smooth crater fills in the lunar equatorial region. It was observed that dielectric constant is a valuable parameter to identify material deposits on the crater floors that indicates the origin of a crater. Craters originating from meteoritic impact and volcanic activities showed different distributions of dielectric constant within their floors. However, such models apply to only smooth, rock-poor regolith surfaces and have limited applications. Consequently, improved algorithms based on physical forward scattering models such as advanced integral equation models (AIEM) have been developed for the estimation of dielectric constant and surface roughness of lunar regolith and other targets. Hence, inversion modelling requirements to retrieve these parameters call for observations in full-polarimetric mode.

\section{Study of geological and morphological features}

The lunar surface is marked with innumerable geological (can be more appropriately called selenological) and morphological features evolved through geological timescale by activities like meteoritic impact, volcanic eruptions and solar winds. Such features carry a lot of information about the origin and evolution of the lunar crust and physical processes acting on the lunar surface. While many of these geological and morphological features have been studied and the associated processes have been understood through optical sensors by previous lunar missions, an orbiting SAR is expected to provide much more details of such features due to its different target interaction mechanisms and surface penetration capability. Many geological and morphological features pertaining to the lunar polar regions were studied and mapped using Chandrayaan-1 Mini-SAR data. However, due to the poor resolution of these data, finer details of the lunar morphological features and many smaller geological features could not be studied ${ }^{13,14}$.

Hence, high spatial resolution and polarimetric data of SAR will enable such features to be studied with finer details of their structure and composition. These studies will lead to generation of high-resolution morphological maps and will facilitate crater age-determination based on size-frequency distribution of smaller craters and secondary craters on the floors of larger craters.

\section{Quantitative estimation of regolith thickness and distribution}

The emissivity corresponding to lunar surface materials in the microwave region as measured by radar brightness temperature $\left(T_{B}\right)$ can provide valuable information about regolith distribution over the lunar surface. The $T_{B}$ as measured at higher wavelengths such as S- and L-bands corresponds to emitted signals from a thick layer depending on the penetration depth at those wavelengths, and is likely to provide information about regolith depth, distribution and its characteristics. Chandrayaan-2 dualfrequency SAR will be operated in passive microwave mode over selected regions in the lunar polar and nonpolar regions, and will provide possible opportunity for quantitative estimation of regolith distribution. Relatively poor $T_{B}$ sensitivity of Chandrayaan-2 SAR radiometer mode $(\sim 6 \mathrm{~K})$ will still be helpful for characterizing regolith thickness and distribution at regional scale.

To summarize, the following are the major science objectives of the Chandrayaan-2 L- and S-band SAR:

(1) Unambiguous detection and quantitative estimation of water-ice deposits in the lunar PSRs, using dualfrequency and full polarimetric imaging.

(2) Dielectric constant and surface roughness estimation over the lunar surface using multi-frequency SAR data.

(3) Study of geological (selenological) evolution processes on the lunar surface, especially in the polar regions through preparation of high-resolution geomorphological maps and crater floor mapping.

(4) Estimation of regolith thickness and distribution at the regional scale using dual-frequency radiometer-mode data.

\section{L- and S-band SAR instrument}

To meet the science requirements, the SAR payload has been designed as two independent and physically separate systems, viz. L-band SAR and S-band SAR, sharing a common antenna. The payload, as a whole, is configured to operate in either standalone (L-band/S-band) or synchronous (L- and S-band) modes of operation. The hallmark of this imaging system is its capability to operate in fully polarimetric mode, which is crucial for unambiguous detection of water-ice. Thus, Chandrayaan-2 L- and S-band SAR will be the first fully polarimetric instrument in a lunar orbit and poised to yield important scientific findings. It is also capable of operating in hybrid polarimetric mode (transmit in circular and receive in linear $\mathrm{H}$ and $\mathrm{V}$ polarizations) and dual-pol mode (transmit in $\mathrm{H}$ and receive in both $\mathrm{H}$ and $\mathrm{V}$ polarizations: $\mathrm{HH}+\mathrm{HV})$.

Full polarimetric mode is generated using interleaved $\mathrm{H}$ and $\mathrm{V}$ transmission, with a pulse-repetition frequency high enough to sample the Doppler spectra sufficiently. This mode allows measurements in all four polarization combinations of $\mathrm{HH}, \mathrm{HV}, \mathrm{VV}$ and $\mathrm{VH}$ and therefore enables extraction of the complete $4 \times 4$ coherency matrix, considered to be important for unambiguous detection of water-ice. However, the mode has significant implications on data rate, data volume and raw bus power, due to which synchronous dual-frequency full polarimetric mode is restricted to narrow pulse width chirp (linear frequency modulation) transmission. Also, 
generally speaking, during full polarimetric operation, high frequency of the receive windows causes reduction in swath or range coverage, or both. In Chandrayaan-2 SAR, L-band full polarimetric and S-band full polarimetric modes are separately available with swath of $10 \mathrm{~km}$ and incidence angle coverage up to $\sim 36^{\circ}$, to be operated with $50 \mu$ s pulse width, while dual-frequency dual polarimetric mode can be operated with maximum pulsewidth of $80 \mu \mathrm{s}$.

In hybrid polarimetric mode, $\mathrm{H}$ and $\mathrm{V}$ polarized signals are transmitted simultaneously with a relative phase offset of $90^{\circ}$, which combine in free space to produce circularly polarized waves ${ }^{15}$. The backscattered signals are received in linear $\mathrm{H}$ and $\mathrm{V}$ polarizations, which are processed to extract the four Stokes parameters, without compromising on swath or range coverage.

The L- and S-band SAR will operate from a $100 \mathrm{~km}$ circular polar orbit of the Moon, mainly in the dawndusk season. Incidence angle coverage from $9.6^{\circ}$ to $36.9^{\circ}$, on either side of the sub-satellite track, will be achieved by roll-tilting the Chandrayaan-2 orbiter. SAR will operate in conventional strip map mode with swath coverage of $10 \mathrm{~km}$ (Figure 2). Slant range resolution is selectable from 2 to $75 \mathrm{~m}$, in eight discrete steps, corresponding to chirp bandwidth of $75-2 \mathrm{MHz}$ respectively. The data acquisition period is typically $8 \mathrm{~min}$ in each $2 \mathrm{~h}$ orbit, primarily during the dawn-dusk seasons.

The hardware configuration of both L- and S-band SAR is broadly similar. The transmit chain comprises a digital chirp-generator that feeds the baseband chirp of required bandwidth to the modulator residing in the frequency-generator unit. The modulated carrier is split into two chains ( $\mathrm{H}$ and $\mathrm{V})$, amplified by the respective solid state power amplifiers (SSPA) to $45 \mathrm{~W}$ (L-band)/

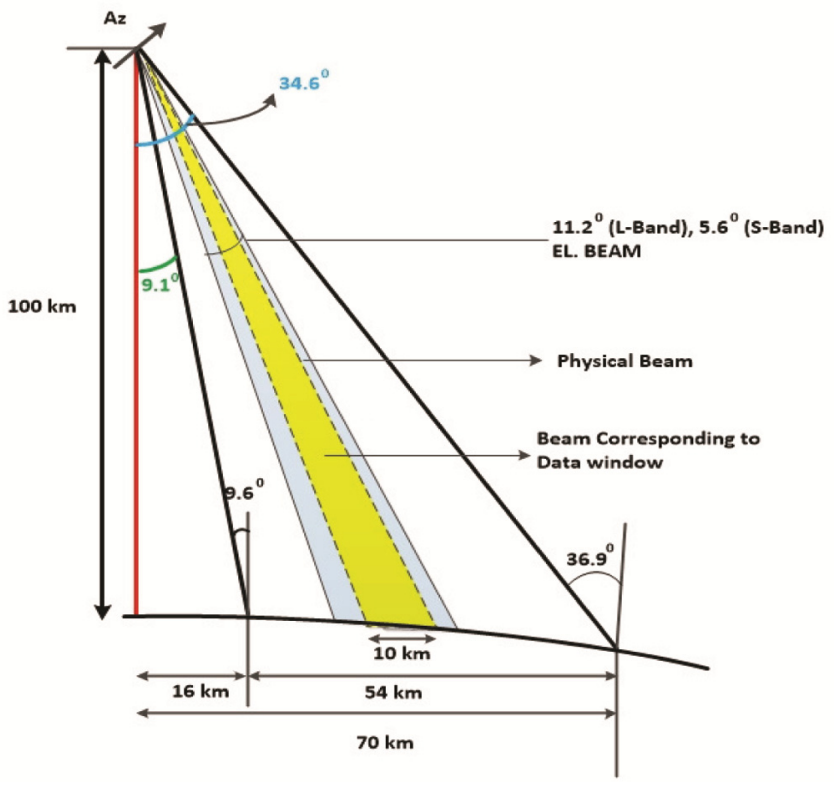

Figure 2. Imaging geometry of Chandrayaan-2 SAR.
$40 \mathrm{~W}$ (S-band) and fed to the antenna at the respective $\mathrm{H}$ and $\mathrm{V}$ ports. The back scattered signals received in $\mathrm{H}$ and $\mathrm{V}$ polarization chains are amplified and demodulated in the receiver. The baseband signals are then digitized by 8-bit analog-to-digital convertors and processed for onboard range compression. The payload data are streamed along with those of other sensors by the spacecraft at appropriate times during earth-visibility periods. Payload controller interfaces with the telemetry and telecommand (TTC) system of the orbiter to receive commands for imaging sessions. These are translated into commands and controls, specific to each payload subsystem and are communicated to them with requisite timings. To achieve synchronous L- and S-band operation, the payload controllers of L- and S-band SAR act as master and slave respectively; thereby, synchronizing the various commanding signals to enable the operation.

The L- and S-band common antenna is designed to cater to S-band TTC requirements of the lander, calling for an extended bandwidth of $180 \mathrm{MHz}$ in the S-band. The selection of functionality is effected by means of two switches provided in $\mathrm{V}$ and $\mathrm{H}$-polarization paths (Figure $3)$. This is possible since the operation of the SAR and communications subsystem are mutually exclusive. A $0^{\circ} / 90^{\circ}$ hybrid is used to convert signals from linear to circular polarization, required for lander TTC.

Chandrayaan-2 SAR is the first mission from Indian Space Research Organisation (ISRO) which has range compression built into the system to contain the data rates to permissible limits. With the imaging geometry of this payload, a significant fraction of the received echo duration is occupied by the pulse width itself; the number varies from $38 \%$ to $70 \%$ depending on transmit chirp pulsewidths and incidence angle. Hence, the digitized baseband receive signals are correlated with chirp replica in the SAR processor to achieve the desired range compression onboard. As the range-compressed data preserve the Gaussian characteristics of the signal, block adaptive quantization (BAQ; 4-bit nominal) is used to further compress the data to meet the rate limit of 160 Mbps.

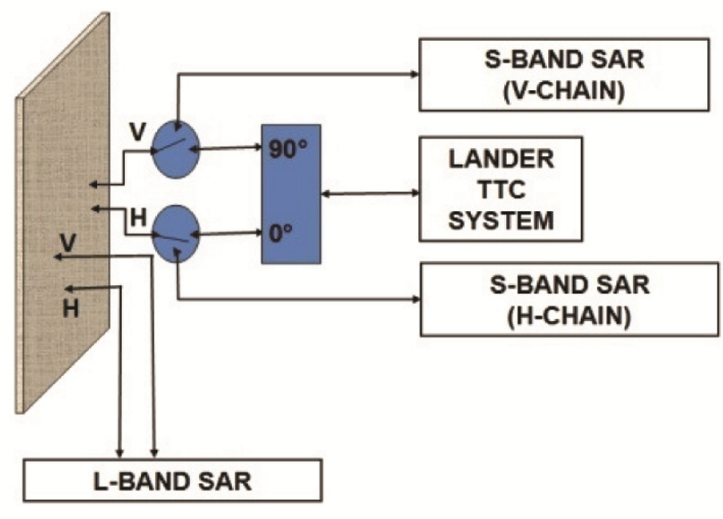

Figure 3. SAR configuration with Lander communication system. 
Table 1. Dual-frequency synthetic aperture radar specifications

\begin{tabular}{|c|c|c|}
\hline & L-Band & S-Band \\
\hline Altitude & \multicolumn{2}{|c|}{$100 \mathrm{~km}$} \\
\hline Frequency & $1.25 \mathrm{GHz}$ & $2.5 \mathrm{GHz}$ \\
\hline SAR modes & \multicolumn{2}{|c|}{ Single/dual/hybrid and full polarimetry } \\
\hline Range swath & \multicolumn{2}{|c|}{$10 \mathrm{~km}$} \\
\hline Incidence angle & \multicolumn{2}{|c|}{$9.6^{\circ}-36.9^{\circ}$} \\
\hline Resolution & \multicolumn{2}{|c|}{$2-75 \mathrm{~m}$} \\
\hline Chirp bandwidth & \multicolumn{2}{|c|}{$75-2 \mathrm{mHz}$ programmable } \\
\hline Antenna & \multicolumn{2}{|c|}{ Microstrip antenna, $1.35 \mathrm{~m} \times 1.1 \mathrm{~m}$} \\
\hline Antenna gain & $22 \mathrm{dBi}$ & $25 \mathrm{dBi}$ \\
\hline Axial ratio in LHCP & $0.4 \mathrm{~dB}$ & $1.1 \mathrm{~dB}$ \\
\hline Transmit pulsewidth & \multicolumn{2}{|c|}{$\begin{array}{c}80-25 \mu \mathrm{s} \text { (hybrid polarimetric) } \\
50-25 \mu \mathrm{s} \text { (full polarimetric) }\end{array}$} \\
\hline SSPA peak power & $45 \mathrm{~W}$ & $40 \mathrm{~W}$ \\
\hline Maximum duty cycle & \multicolumn{2}{|l|}{$24 \%$} \\
\hline Receiver noise figure & $2.8 \mathrm{~dB}$ & $4.3 \mathrm{~dB}$ \\
\hline $\begin{array}{l}\text { Noise equivalent sigma-naught at swath end } \\
\text { for } 75 \mathrm{~m} \text { resolution and } 30^{\circ} \text { incidence }\end{array}$ & $\begin{array}{l}-33.7 \mathrm{~dB} \text { (hybrid polarimetric) } \\
-30 \mathrm{~dB} \text { (full polarimetric) }\end{array}$ & $\begin{array}{l}-26.2 \mathrm{~dB} \text { (hybrid polarimetric) } \\
-22.5 \mathrm{~dB} \text { (full polarimetric) }\end{array}$ \\
\hline Onboard processing & \multicolumn{2}{|c|}{ Range compression and BAQ } \\
\hline Data rate & \multicolumn{2}{|c|}{$160 \mathrm{Mbps}$} \\
\hline Raw bus power & \multicolumn{2}{|c|}{$100 \mathrm{~W}$} \\
\hline Payload mass & \multicolumn{2}{|c|}{$20 \mathrm{~kg}$} \\
\hline
\end{tabular}

A fraction of the transmitter output is coupled directly to the receiver for chirp-replica acquisition to enable range compression. A back-to-back 1:2 combiner/divider network combines $\mathrm{H}$ and $\mathrm{V}$ coupled signals first and then divides equally before feeding to the coupled ports of the receivers. This is necessary for acquiring the replica in all the four polarization combinations of $\mathrm{HH}, \mathrm{HV}, \mathrm{VV}$ and $\mathrm{VH}$, and is critical for polarimetric applications. Every imaging session is preceded and followed by a set of replica acquisitions. A dedicated mode for calibration is also configured in the system.

One of the important requirements for polarimetric SAR is to ensure minimum amplitude and phase imbalances between $\mathrm{H}$ and $\mathrm{V}$ chains of transmitter as well as receiver. The transmitters operate in saturation and hence are expected to have a good amplitude balance; however, phase biases and imbalances are expected and are required to be corrected. To do so, each chain of $\mathrm{H}$ and $\mathrm{V}$ transmitters is provided with 6-bit digital phase shifters, to correct the phase in steps of $5.625^{\circ}$. Characterization tests of the integrated transmit chain reveal the exact phase bias and imbalance numbers. These measurements are used to arrive at the optimal settings for fully balanced phases of $\mathrm{H}$ and $\mathrm{V}$, required for full polarimetric mode of operation. For hybrid polarimetry mode, additional phase shift of $90^{\circ}$ in one of the transmit chains is applied to generate circularly polarized transmission. These phase shifts are applied after compensating for temperature-induced variations using characterization data held in look-up tables in the payload controller. For the receive chains, imbalances are measured during ground characterization and are taken care of during processing.
Provision has been made in the SAR system to operate in radiometer mode. It has been added at a late stage of system realization, by implementing updates only in controller software and processor, without any implications on the hardware design and configuration. During the radiometer-mode operation, the RF transmission is inhibited and protection switches (absorptive in nature) at the receiver input are toggled to get two sets of data-one, 'noise only' when antenna path is isolated by the switch and two, 'signal + noise' when antenna path is connected. The measurements are averaged for data window duration onboard and downlinked for further averaging on-ground to improve temperature sensitivity. It is important to note that measurements by this mode are not equivalent to those with a conventional radiometer, as there are a number of design differences between receive chains of SAR and radiometer, including that of antenna patterns and calibration requirements. Hence, in-orbit measurements in radiometer mode will be carried out on an experimental basis to effectively use the sensor during the periods when SAR is not in operation. Prior to the start of the radiometer-mode operations, the spacecraft will be yaw-rotated by $110^{\circ}$ for deep-sky cold calibration to improve the accuracy of $T_{B}$ estimates.

Table 1 enlists the system specifications in brief. The radiometric performance of SAR is measured in terms of noise equivalent sigma naught (NESZ) or equivalently, the lowest backscatter coefficient detectable by the system. Only the worst-case NESZ value corresponding to $75 \mathrm{~m}$ slant-range resolution is presented in the table, whereas Figure 4 shows how the values vary over swath and range of incidence angles. Radiometric resolution (with 18-look processing) has been estimated to be $1.7-1 \mathrm{~dB}$, 
RESEARCH ARTICLES
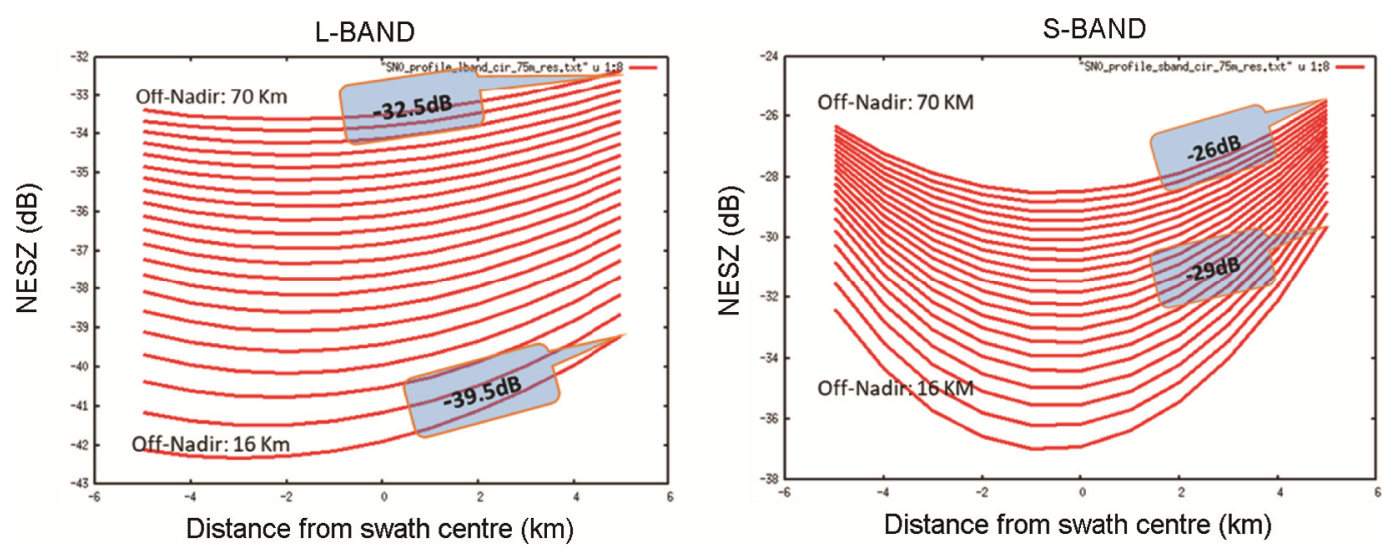

Figure 4. Noise equivalent sigma naught (NESZ) for L- and S-band SAR systems corresponding to hybrid polarimetry mode with $2 \mathrm{mHz}$ bandwidth.

for a practical range of target backscatter properties resulting in signal-to-noise ratios (SNRs) of $0-10 \mathrm{~dB}$ respectively. SAR radiometric accuracy is specified to be better than $1 \mathrm{~dB}$.

To meet the limited resource allocations typical for an inter-planetary mission, a number of measures have been taken during hardware realization for miniaturization and high efficiency. The antenna is highly optimized for mass and weighs a mere $3.6 \mathrm{~kg}$, while meeting the performance requirements. The SSPAs of the transmitters are realized using efficiency enhancement techniques to achieve a high efficiency of the order of $45 \%$, critical to meet $100 \mathrm{~W}$ raw-power allocation to SAR. Indigenously developed microwave integrated circuits, monolithic microwave integrated circuits and multichip modules have been used extensively in the receiver to achieve the mass and volume targets.

All the digitally implemented functionalities in the payload, viz. chirp generation, control and timing signal generation, synchronization between L- and S-band SAR systems during simultaneous operations, data acquisition and onboard compression have been integrated into a single-board high-density entity using field programmable gate array devices (FPGA, Xilinx Virtex 4) and applicationspecific integrated circuits (ASIC), thereby achieving significant gain in mass and volume.

To enable sufficient isolation between the L- and Sbands and to reject other out-of-band signals, front-end of the receivers has been equipped with filters. Though this has penalty of receiver noise figure (and hence, system SNR), it is critical for the safety of S-band SAR, due to the constantly present S-band TTC frequencies (communication link to the Earth) onboard.

All subsystems of the L- and S-band SAR payload have been realized and integrated as a payload at Space Applications Centre (SAC), ISRO, Ahmedabad (Figure 5). The instrument has been integrated with the Chandrayaan-2 orbiter spacecraft and has undergone all tests successfully.

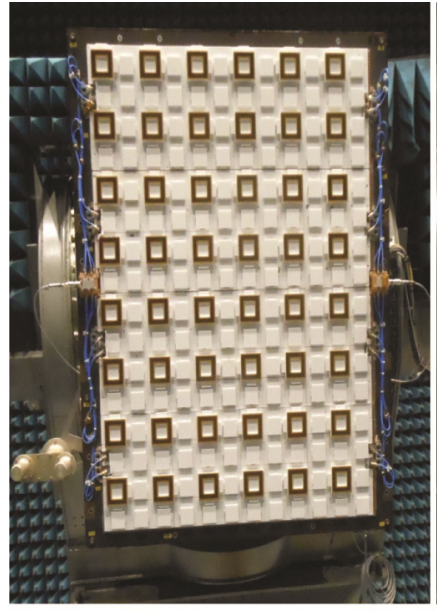

Dual frequency SAR antenna

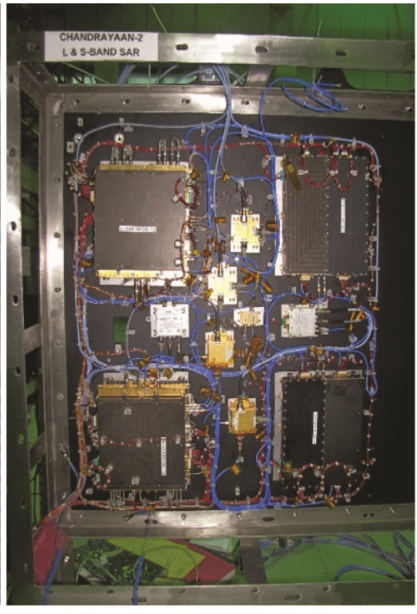

Dual frequency SAR electronics
Figure 5. Integrated flight hardware of L- and S-band SAR in payload integration laboratory.

\section{Instrument characterization}

In addition to the rigorous payload testing for various operating conditions, an exhaustive characterization is the key to achieve the desired science results. This is more so in the case of a polarimetric SAR. Hence, Chandrayaan-2 SAR payload has been tested and characterized thoroughly for polarimetric performance at payload and spacecraft levels.

Antenna gain patterns have been measured in far-field using compact antenna test facility at SAC. The antenna gains along with transmit peak power and receive-path noise-figure measurements are part of the fundamental yet important set of measurements to estimate NESZ of the SAR payload (Table 1).

Internal calibration using optical delay line-based setup $^{16}$, was adopted to test the payload end-to-end in a fully-integrated condition. Using this, impulse responses were measured for different bandwidths in an operational 
scenario. Performance of onboard range compression was tested by feeding simulated SAR raw data to the digital processor of the payload. The receiver chain has been characterized to map the digital output to the input signal levels.

Chirp signals through coupled ports of transmitter and receiver ('chirp replicas') were measured in calibration mode along with transmit peak power. With this, any variation of combined gain of transmitter and receiver can be tracked. Noise-power measurements when transmit pulses are off enable tracking of receiver gain. It is important to note that the payload imaging sessions begin with acquisitions of the chirp replica and noise measurement.

Simultaneous operation of L- and S-band SAR has been demonstrated with phases synchronized by $6^{\circ}$ or better. This is a notable achievement considering the fact that both frequency bands are generated using different oscillators, running independently and located in the respective frequency-generator units. This was possible due to the data acquisition scheme of first capturing the chirp replica prior to start of actual imaging, and using it for range compression. This way, the starting phases of the transmitted chirps are effectively compensated.

Axial ratio measurements were done for left- and righthanded circularly polarized (LHCP/RHCP) transmit chains. Optimal phase combinations of the transmitter phase shifters were derived for LHCP and RHCP in this process. Measurements for amplitude and phase imbalances and cross talk between $\mathrm{H}$ and $\mathrm{V}$ chains of transmitter and receiver were done at integrated payload level and re-verified at integrated spacecraft level.

Radiometer-mode measurements were carried out during ground characterization tests using blackbody target (RF absorbers at laboratory ambient temperature). This is equivalent to hot-load calibration of the radiometer. The temperature sensitivity has been estimated to be $\sim 6 \mathrm{~K}$, which is as expected, considering the fact that the system was not originally designed as a conventional radiometer and basically, makes use of SAR receive chain only for noise measurements.

\section{Post-launch calibration and validation}

Absolute calibration of the instrument is necessary to convert the pixel value of the image into an interpretable physical parameter, which is backscatter coefficient (sigma naught) in the case of SAR. For Earth-observing SAR missions, absolute calibration is done using corner reflectors and active radar calibrators of known RCS. However, this method cannot be used for planetary SARs due to absence of such standard targets on the planetary surface. The method adopted for Chandrayaan-2 SAR makes use of characterization data of the receive path, wherein digital output was mapped to input signal level, clubbed with measured values of transmit power and antenna gain. Absolute calibration is planned, postlaunch, during commissioning phase of the instrument. For the S-band SAR, verification of the absolute calibration is planned using S-band Mini-RF sigma-naught values, by imaging over regions of extended smooth surfaces, mostly pyroclastic deposits, in the equatorial regions of the Moon. However, as this is the maiden L-band SAR in a lunar orbit, such inter-sensor calibration will not be possible in the L-band.

Relative calibration of $\mathrm{H}$ and $\mathrm{V}$ chains, carried out during ground characterization, will be validated by imaging in nadir-view geometry over smooth, flat and radar-dark (low sigma naught) regions of the Moon. By doing so, the amplitudes of $\mathrm{HH}$ and $\mathrm{VV}$ polarized signals are expected to be equal, and the relative phase between them to be negligible. Any disparity observed may be measured and compensated during ground-processing. This experiment will help in polarimetric calibration of SAR data by offsetting any deviation in amplitude and relative phase observed in $\mathrm{HH}$ and VV polarized signals.

For radiometer-mode operations, two-point calibration using low and high $T_{B}$ measurements is necessary to map the digital output to input $T_{B}$. For this, cold calibration will be done by orienting the spacecraft for SAR antenna to view the deep sky $\left(T_{B}\right.$ of $\left.2.7 \mathrm{~K}\right)$. There is no provision of hot-load calibration onboard. Hence, for hot-load calibration in integrated mode, ground measurements with RF blackbody will be used. Additionally, receiver gain variations in orbit can be tracked by noise-only measurements (receiver input in terminated condition) made at every alternate data-window in radiometer mode.

\section{Operation plan for science data}

The science objectives and corresponding modes of operation have been defined. Both poles of the Moon between $80^{\circ}$ and $90^{\circ}$ lat. will be imaged as priority using the Lband full polarimetric mode. Data will be processed with appropriate decomposition technique to identify regions with unambiguous presence of water-ice. The data will also be used for estimation of dielectric constant and surface roughness over these identified regions. The regions will be mapped by simultaneous L- and S-band imaging in dual polarization $(\mathrm{HH}+\mathrm{HV})$ for LPDI computation. The LPDI indices, and estimated dielectric constant and surface roughness will be used for volumetric estimation of water-ice. The total coverage period for both the poles is expected to be about four months using the culling approach for the highly overlapping regions in the poles.

High-resolution imaging over identified regions of the Moon is planned next to study geological (selenological) evolution processes on the lunar surface, especially in the polar regions through preparation of high-resolution geomorphological maps and crater floor-mapping. This will 
be followed by dielectric constant and surface roughness estimation using full polarimetric mode.

The allocated SAR imaging period is $8 \mathrm{~min}$ per $2 \mathrm{~h}$ orbit in the dawn-dusk season. The non-imaging periods of SAR can be used for radiometer mode, which is a lowpower mode and easy on spacecraft resources. However, the windows for radiometer-mode operation will be planned by the mission keeping in view operation of other sensors onboard Chandrayaan-2. $T_{B}$ data from the radiometer mode will help in quantitative estimation of regionalscale regolith thickness and distribution ${ }^{17}$.

\section{Summary}

L- and S-band polarimetric SAR for Chandrayaan-2 has been configured to address diverse requirements of the scientific community, with specific modes for high resolution and high radiometric sensitivity. Unanswered questions and ambiguities from the previous missions, mainly relating to water-ice detection are attempted to be resolved using dual frequency SAR in full-polarimetric mode of imaging. The system design efficiently marries the science requirements and the demanding spacecraft constraints, to pack in various features to maximize performance in the resource-constrained environment of a planetary mission. The SAR system is expected to significantly contribute towards the enhancement of knowledge about lunar polar volatiles, surface and subsurface features.

1. Spudis, P. D. et al., MiniSAR: an imaging radar experiment for Chandrayaan-1 mission to the Moon. Curr. Sci., 2009, 96(4), 533539.

2. Spudis, P. D. et al., Initial results for the North Pole of the Moon from Mini-SAR, Chandrayaan-1 mission. Geophys. Res. Lett., 2010, 37, L06204; doi:10.1029/2009GL042259.

3. Mohan, S., Das, A. and Chakraborty, M., Investigation of polarimetric properties of lunar surface using Mini-SAR data. Curr. Sci., 2011, 101(2), 159-164.

4. Mohan, S., Saran, S. and Das, A., Scattering mechanism-based algorithm for improved mapping of water-ice deposits in the lunar polar regions. Curr. Sci., 2013, 105(11), 1579-1587.

5. Pieters, C. M. et al., Character and spatial distribution of $\mathrm{OH} / \mathrm{H}_{2} \mathrm{O}$ on the surface of the Moon seen by M3 on Chandrayaan-1, Science, 2009, 326, 568-572.
6. Spudis, P. D. et al., Evidence for water ice on the Moon: results for anomalous polar craters from the LRO Mini-RF imaging radar, J. Geophys. Res.: Planets, 2013, 118, 2016-2029.

7. Mishra, P., Kumar, S. and Singh, D., An approach for finding possible presence of water ice deposits on Lunar craters using MiniSAR data. IEEE J. Sel. Topics Appl. Earth Obs. Remote Sensing, 2015, 8(1), 30-38.

8. Fa, W., Wieczorek, M. A. and Heggy, E., Modeling polarimetric radar scattering from the lunar surface: study on the effect of physical properties of the regolith layer. J. Geophys. Res., 2011, 116, E03005; doi:10.1029/2010JE003649.

9. Pandey, D., Saran, S., Das, A. and Chakraborty, M., A simplistic approach to model radar backscatter from lunar regolith. In 44th Lunar and Planetary Science Conference (LPSC), Abstr. 1941, 2013.

10. Pandey, D., Das, A., Saran, S. and Chakraborty, M., Scattering characteristics of lunar regolith with respect to dual-frequency SAR: preliminary simulation results. In LPSC XLIV, Abstr. 126, 2013.

11. Putrevu, D., Das, A., Vachhani, J. G., Trivedi, S. and Misra, T., Chandrayaan-2 Dual-frequency SAR: further investigation into lunar water and regolith. Adv. Space Res., 2016, 57, 627-646.

12. Campbell, B. A., Grant, J. A. and Maxwell, T., Radar penetration in Mars analog environments. In LPSC XXXIII, Abstr. \#1616, 2002.

13. Bell, W. S., Thomson, B. J., Dyar, M. D., Neish, C. D., Cahill, J. T. S. and Bussey, D. B. J., Dating small fresh lunar craters with Mini-RF radar observations of ejecta blankets. J. Geophys. Res., 2012, 117, E00h30.

14. Desai J. Ami, Mohan, S. and Murty, S. V. S., Impact ejecta characterization for small-sized fresh and degraded lunar craters using radar data. Curr. Sci., 2016, 110(10), 1929-1938.

15. Raney, R. K., Hybrid-polarity SAR architecture. IEEE Trans. Geosci. Remote Sensing, 2007, 45, 3397-3404.

16. Chan, Y. K., Chung, B. K. and Chuah, H. T., Transmitter and receiver design of an experimental airborne synthetic aperture radar sensor. Progress. Electromagn. Res., 2004, 49, 203-218.

17. Vijayan, S., Mohan, S. and Murty, S. V. S., Lunar regolith thickness estimation using dual frequency microwave brightness temperature and influence of vertical variation of $\mathrm{FeO}+\mathrm{TiO}_{2}$. Planet. Space Sci., 2015, 105, 123-132.

ACKNOWLEDGEMENTS. We acknowledge the dedicated efforts of the entire team of Chandrayaan-2 SAR and all facilities at Space Applications Centre (SAC), Ahmedabad, without which successful realization of the payload would not have been possible. We thank the Director, SAC for constant encouragement and guidance, and reviewers of various committees for their critical comments and suggestions to add more value to the payload and its science.

Received and accepted 27 August 2019

doi: $10.18520 / \mathrm{cs} / \mathrm{v} 118 / \mathrm{i} 2 / 226-233$ 